\title{
Nivel de satisfacción académica en estudiantes de Paramédico y Protección Civil de la Universidad Tecnológica del Valle de Toluca
}

\author{
Level of Academic Satisfaction in Paramedic and Civil Protection Students of \\ the Universidad Tecnológica del Valle de Toluca
}

\begin{abstract}
Nível de satisfação acadêmica de estudantes de Paramédico e Proteção Civil da Universidade Tecnológica de Valle de Toluca
\end{abstract}

Fabiola Rivera Ramírez

Universidad Tecnológica del Valle de Toluca, México

fabiola.rivera@utvtol.edu.mx http://orcid.org/0000-0001-7296-1378

\section{Resumen}

La satisfacción académica es un indicador de calidad. Para la Universidad Tecnológica del Valle de Toluca implica una mayor motivación, sentimiento de pertinencia, permanencia y una menor deserción. Por lo que el objetivo de este trabajo fue indagar el nivel de satisfacción académica en los estudiantes tanto de Técnico Superior Universitario en Paramédico Escolarizado (TSUPE) como despresurizada (TSUPD) y de la Licenciatura en Protección Civil y Emergencia (LPCE) de dicha institución educativa.

Para ello se adaptó y aplicó el instrumento para evaluar la satisfacción del estudiante diseñado por Álvarez, Chaparro y Reyes (2015) a 320 alumnos, clasificados por grado académico. Asimismo, se realizó un análisis de varianza, una correlación de Pearson y un análisis de componentes principales $(p<0.05)$.

Los resultados indican que los alumnos de TSUPE, TSUPD y LPCE se encuentran satisfechos con la calidad académica. Y que la atención por parte del docente es el principal factor. Por último, un aspecto de mejora son los servicios de apoyo. 


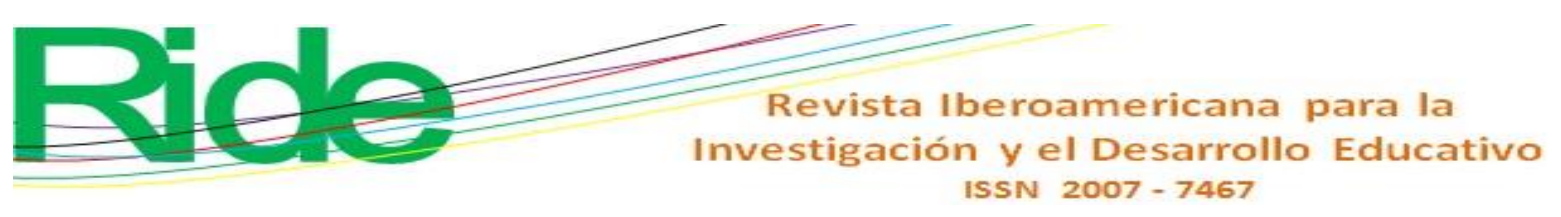

Palabras clave: atención prehospitalaria, educación universitaria, Licenciatura en Protección Civil, Técnico Superior Universitario en Paramédico, satisfacción académica.

\section{Abstract}

Academic satisfaction is an indicator of quality. For the Universidad Tecnológica del Valle de Toluca it implies a higher motivation, feeling of relevance, permanence and a lower dropout. Therefore, the aim of this work was to investigate the level of academic satisfaction in students of both Higher University Technician in School Paramedics (TSUPE) and depressurized (TSUPD) and Bachelor's in Civil Protection and Emergency (LPCE) of said educational institution.

To reach the above mentioned the instrument to evaluate student satisfaction designed by Álvarez, Chaparro and Reyes (2015) was adapted and applied to 320 students, classified by academic degree. Likewise, a variance analysis, a Pearson correlation, and a principal component analysis $(p<0.05)$ were performed.

The results indicate that TSUPE, TSUPD and LPCE students are satisfied with academic quality, with the teacher's attention being the main factor. One aspect of improvement is support services.

Keywords: prehospital care, university education, Degree in Civil Protection, Advanced University Technician in Paramedic, academic satisfaction.

\section{Resumo}

A satisfação acadêmica é um indicador de qualidade. Para a Universidade Tecnológica do Vale do Toluca, isso implica em maior motivação, sentimento de relevância, permanência e menor abandono. Portanto, o objetivo deste trabalho foi investigar o nível de satisfação acadêmica dos estudantes de ambos os Técnicos Universitários em Paramédicos Escolares (TSUPE) e despressurizados (TSUPD) e o Grau de Proteção Civil e de Emergência (LPCE) da referida instituição educacional.

Para tanto, o instrumento foi adaptado e aplicado para avaliar a satisfação do aluno de Álvarez, Chaparro e Reyes (2015) a 320 alunos, classificados por grau acadêmico. Da mesma forma, foram realizadas análises de variância, correlação de Pearson e análise dos principais componentes $(\mathrm{p}<0,05)$. 


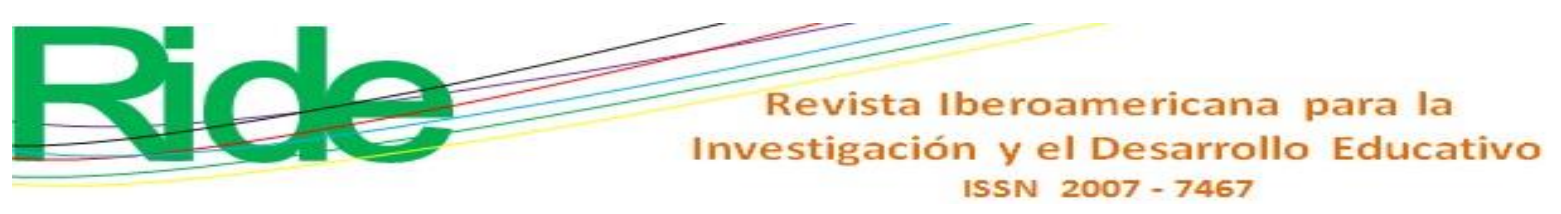

Os resultados indicam que os alunos do TSUPE, TSUPD e LPCE estão satisfeitos com a qualidade acadêmica. E que a atenção do professor é o principal fator. Finalmente, um aspecto da melhoria são os serviços de suporte.

Palavras-chave: atendimento pré-hospitalar, educação universitária, Bacharel em Proteção Civil, Técnico Universitário de Paramédicos, satisfação acadêmica.

Fecha Recepción: Abril 2019

Fecha Aceptación: Octubre 2019

\section{Introducción}

La educación en la atención prehospitalaria ha evolucionado: de la formación informal a la universitaria (Chang, Tsai y Williams, 2017; Devenish, 2014; Hou, Rego y Service, 2013). Actualmente, en México se utiliza la certificación de grado o profesionalización (título y cédula profesional). Al respecto, la Universidad Tecnológica del Valle de Toluca (UTVT) cuenta con los grados de Técnico Superior Universitario en Paramédico (TSUP) en modalidad escolarizada (TSUPE) y despresurizada ${ }^{1}$ (TSUPD), con su continuidad en la Licenciatura en Protección Civil y Emergencias (LPCE).

Sin embargo, como sucede en otras disciplinas médicas, los programas universitarios para la atención prehospitalaria son una mezcla de educación práctica y científica, por lo que existen diversos factores que pueden influir en el proceso de aprendizaje y satisfacción (Hou et al., 2013; Osorio y Parra, 2016).

La satisfacción estudiantil con la formación recibida se define como el estado de ánimo que poseen los estudiantes con respecto a su institución. Es resultado de la percepción del cumplimiento de sus necesidades, expectativas y requisitos (Agustín y Domelis, 2009). Confirma, además, la calidad educativa y refleja la eficiencia de los servicios académicos y administrativos; lo que implica una mayor motivación, sentimiento de pertenencia, permanencia y una difusión positiva de la actividad académica (González, Pino y Penado, 2017; Oliva y Gómez, 2014; Pérez y Pereyra, 2015; Silva, Domínguez, Cortés, Castorena y Polo, 2015).

\footnotetext{
${ }^{1}$ La modalidad despresurizada es un sistema para aquellas personas que desean continuar con sus estudios universitarios y que por motivos de trabajo, diversidad de horarios en sus actividades personales $u$ otras razones no pueden integrarse a un horario escolar en días hábiles.
} 


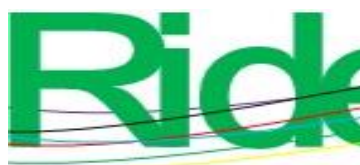

Revista Iberoamericana para la
Investigación y el Desarrollo Educativo
ISSN $2007-7467$

La razón de medirla se fundamenta en el hecho de que los estudiantes son el eje nuclear y la garantía de la existencia y mantenimiento de las organizaciones educativas. Ellos, como destinatarios de la educación, son los que mejor pueden valorarla y, aunque presenten una visión parcial y subjetiva, su opinión proporciona un referente que debe tomarse en cuenta. Medir la satisfacción del alumno tiene sentido siempre que vaya acompañada de acciones que promuevan la mejora y la innovación; es por ello por lo que medir dicho constructo de una manera consistente, permanente y adecuada orientaría a la toma de decisiones correctas, con la intención de aprovechar las oportunidades de mejora (González et al., 2017).

La satisfacción es trascendental para valorar un servicio, hasta el punto de marcar diferencias en el mercado. En el caso de la educación, puede influir en el deseo del estudiante de asistir a la universidad o de desertar, por lo que se presenta como la mejor estrategia para evitar el abandono; está influenciada por la confianza, de modo que se puede crear mediante un trato equitativo hacía con los alumnos, cumpliendo sus expectativas y manejando las quejas de modo eficaz (Alonso Dos Santos, 2016).

Por tal motivo, es importante que los estudiantes manifiesten su opinión acerca de las unidades de aprendizaje, las interacciones que tienen con su profesor, compañeros de clase y personal administrativo, y de las instalaciones, equipo y servicios proporcionados por la institución (Silva et al., 2015). El presente estudio tiene como objetivo indagar a través de la encuesta propuesta por Álvarez, Chaparro y Reyes (2015) el nivel de satisfacción académica en los estudiantes de la carreras de TSUPE, TSUPD y LPCE de la UTVT.

\section{Método}

Se trata de un estudio transversal, descriptivo y correlacional, realizado en estudiantes de TSUPE, TSUPD y LPCE, pertenecientes a la Dirección de Carrera de Protección Civil de la UTVT, ubicada en la población de Santa María Atarasquillo, en el municipio de Lerma, Estado de México.

Para la elección del instrumento de evaluación se realizó una revisión bibliográfica. A partir de esta, se seleccionó y adaptó el propuesto por Álvarez et al. (2015), que mide, mediante una escala tipo Likert de cinco valores $(1=$ Totalmente insatisfecho, $2=$ Insatisfecho, 3 = Satisfecho, 4 = Muy satisfecho, 5 = Totalmente satisfecho), ocho 


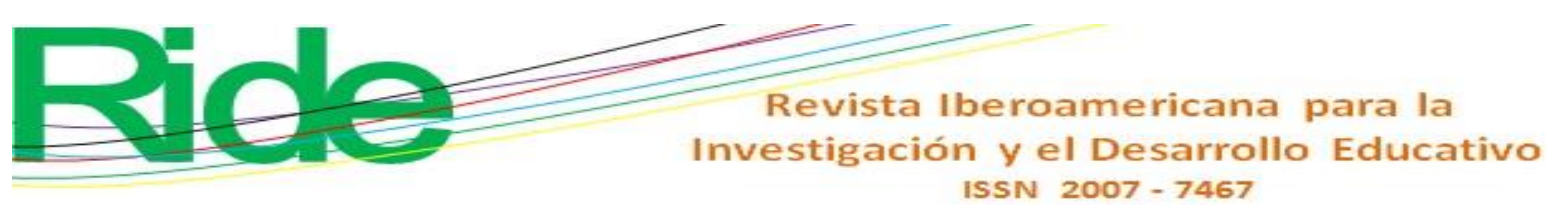

indicadores que intervienen en la satisfacción de los alumnos: Plan de estudios (PE), Capacitación y habilidad para la enseñanza de los docentes (CHED), Métodos de enseñanza y evaluación (MEE), Nivel de autorrealización del estudiante (NAE), Servicios de apoyo (SA), Servicios administrativos (SA), Ambiente propicio (AP) e Infraestructura (I).

La recolección de los datos se llevó a cabo durante los meses de marzo y abril del 2018. Fue obtenida con la ayuda de la herramienta Formularios de Google. Y se empleó un muestreo aleatorio simple. Los criterios de inclusión se enuncian a continuación: género indistinto, inscritos en algunas de las carreras antes mencionadas y que aceptaran participar en el estudio.

Los datos recolectados se exportaron a una hoja de trabajo del programa Microsoft Excel, para su posterior análisis estadístico con el programa Sigma Plot versión 13.0 y el complemento estadístico para Excel Real Statistics. Las variables cuantitativas se representaron con medidas de tendencia central (media y mediana) y de dispersión (desviación estándar [DE]) y las cualitativas en porcentajes y frecuencias absolutas. Se realizó el análisis de confiabilidad con la determinación del coeficiente alfa de Cronbach, cuyos valores aceptables son mayores a 0.7 y de excelencia, arriba de 0.9.

Asimismo, para el análisis estadístico de los datos obtenidos, estos se clasificaron por género y grado escolar (LPCE, TSUPE y TSUPD). Se utilizó la prueba de la ji al cuadrado para comprobar si las puntuaciones en los indicadores de satisfacción eran diferentes atendiendo a la clasificación antes mencionada. Posteriormente, se realizó la comparación de dos o más medias empleando el análisis de varianza de un solo factor (Anova) (pruebas post-hoc de Kruskal Wallis; método de Dunn en caso de resultar significativos) y una $\mathrm{t}$ de Student para muestras independientes, considerando la significancia estadística con un valor de $p<0.05$.

El grupo que presentó significancia estadística con $p<0.05$ (Anova y t de Student) fue el grado escolar. Se utilizó el promedio general obtenido de 3.5 como punto de corte para establecer si los alumnos se encuentran satisfechos (mayor) o insatisfechos (menor).

Por medio del coeficiente de correlación de Pearson, mientras tanto, se analizó la correlación entre los factores de satisfacción, considerando correlaciones muy fuertes mayores de 0.8 , fuertes entre 0.6 y 0.8 , moderadas de 0.4 a 0.6 , débiles entre 0.2 y 0.4 y menores de 0.2 como muy débiles y una $p<0.05$. 

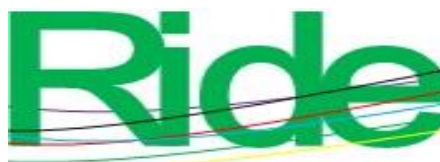

Revista Iberoamericana para la Investigación y el Desarrollo Educativo ISSN $2007-7467$

Por último, se realizó un análisis de componentes principales (ACP). Antes de su ejecución se verificó mediante la prueba de Kaiser-Mayer-Olkin (KMO), cuyo valor debe ser mayor a 0.5. El ACP es una técnica multivariada que permite examinar la relación existente entre diversas variables cuantitativas y puede ser usada para reducir el número de variables. Las combinaciones lineales son ortogonales y en conjunto explican la variabilidad de los datos; el primer componente es el que explica la mayor parte de esta.

\section{Resultados}

\section{Características del alumnado}

De los 412 estudiantes que conformaban la matrícula de la Dirección de Protección Civil y Emergencias de la UTVT durante el cuatrimestre enero-abril 2018, solo 320 contestaron la encuesta, lo que corresponde a $78 \%$ del alumnado. De manera desglosada, $61.25 \%$ (196) son TSUPE: $50.31 \%$ (161) son hombres, con edad promedio de $22.71 \pm$ 5.74 años. También este género obtuvo el mayor porcentaje en el caso de TSUPD, con 75 $\%$ (36) y $31.94 \pm 6.1$, respectivamente (tabla 1 ).

Además, $50.94 \%$ (163) de la totalidad de los estudiantes se dedica a estudiar. Por otro lado, $24.69 \%$ (79) trabajan en el área prehospitalaria. Este es el grupo con mayor porcentaje de entre los que se encuentran en la modalidad despresurizada (TSUPD), con $93.75 \%$ (45) (véase tabla 1). 
Tabla 1. Características de los estudiantes de TSUPE, TSUPD, y su continuidad con la

LPCE en porcentajes

\begin{tabular}{|c|c|c|c|c|c|c|c|c|}
\hline & \multicolumn{2}{|c|}{ LPCYE } & \multicolumn{2}{|c|}{ TSUPE } & \multicolumn{2}{|c|}{ TSUPD } & \multicolumn{2}{|c|}{ Total } \\
\hline Variable & $\begin{array}{c}\text { n/ } \\
\text { media }\end{array}$ & $\begin{array}{l}\% / \\
\text { DE }\end{array}$ & $\begin{array}{c}\mathbf{n} / \\
\text { media }\end{array}$ & $\begin{array}{l}\% / \\
\text { DE }\end{array}$ & $\begin{array}{c}\mathbf{n} / \\
\text { media }\end{array}$ & $\begin{array}{l}\% / \\
\text { DE }\end{array}$ & $\begin{array}{c}\text { n/ } \\
\text { media }\end{array}$ & $\begin{array}{l}\% / \\
\text { DE }\end{array}$ \\
\hline n $(\%)$ & 76 & 23.75 & 196 & 61.25 & 48 & 15 & 320 & 100 \\
\hline Hombres & 32 & 42.11 & 93 & 47.45 & 36 & 75 & 161 & 50.31 \\
\hline $\begin{array}{l}\text { Edad (media } \quad \pm \\
\text { DE) }\end{array}$ & 22.79 & 2.85 & 20.44 & 3.98 & 31.94 & 6.1 & 22.71 & 5.74 \\
\hline \multicolumn{9}{|c|}{ Trabajan } \\
\hline No (solo estudian) & 39 & 51.32 & 123 & 62.76 & 1 & 2.08 & 163 & 50.94 \\
\hline $\begin{array}{l}\text { Área afín o } \\
\text { prehospitalaria }\end{array}$ & 22 & 28.95 & 12 & 6.12 & 45 & 93.75 & 79 & 24.69 \\
\hline Otro & 15 & 19.74 & 61 & 31.12 & 2 & 4.17 & 78 & 24.38 \\
\hline
\end{tabular}

*Edad expresada en media y DE

Fuente: Elaboración propia

\section{Satisfacción académica de los estudiantes de TSUPE, TSUPD y LPCE}

La confiabilidad del instrumento de evaluación de satisfacción académica universitaria fue excelente con 0.96 , determinada mediante el coeficiente de alfa de Cronbach.

En la figura 1 se muestran los porcentajes de satisfacción de acuerdo con la escala de Likert. De manera general, se observa que $35.0 \%$ y $26.3 \%$ se encuentran entre las respuestas de muy satisfechos y satisfechos, mismo fenómeno que se repite en los estudiantes de LPCE (36.6 \% y 25.7\%) y TSUPE (37.4 \% y $28.6 \%$ ). Mientras que para los de TSUPD, está entre totalmente satisfechos (51.4\%) y muy satisfechos $(22.7 \%)$. Es de destacar que los alumnos de LPCE tienen el mayor porcentaje de totalmente insatisfechos $(8.8 \%)$. 


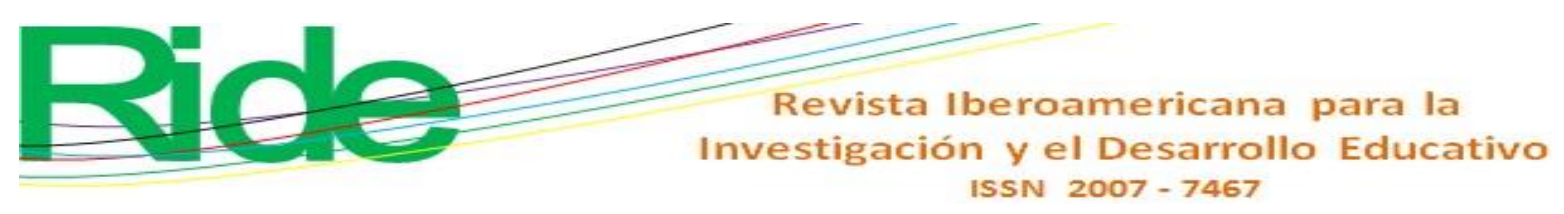

Figura. 1. Porcentajes de satisfacción de acuerdo con las respuestas de los estudiantes encuestados

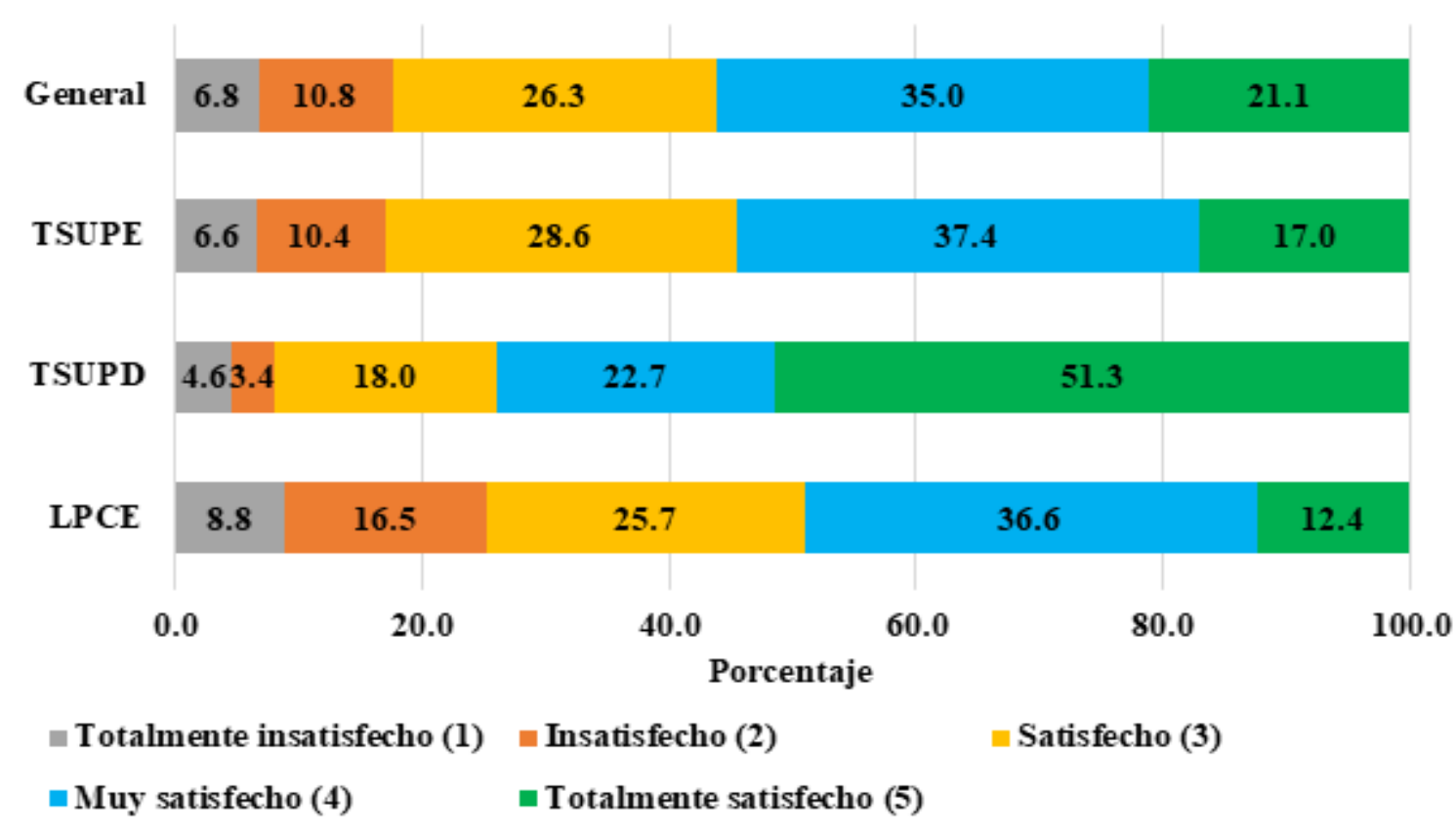

Fuente: Elaboración propia

Posteriormente, se calculó el promedio general y el porcentaje de satisfacción de calidad académica de los estudiantes de las carreras de TSUP y LPCE (ver tabla 2, en donde a la izquierda se encuentra el promedio; a la derecha, el porcentaje de satisfacción), y se obtuvo $3.53 \pm 0.74$ de 5 puntos (figura 1). Por lo que los estudiantes se encuentran satisfechos con la educación recibida. Este valor se empleó como punto de corte para clasificar el nivel de satisfacción, considerando aquellos que están por encima de 3.5 como satisfechos, lo que corresponde a $53.13 \%$ (170).

El puntaje más alto se observó en los estudiantes de TSUPD, con $4.16 \pm 0.84$ ( $p<$ 0.001), y un porcentaje de satisfacción de $81.25 \%$ (39), lo que indica que estos alumnos están muy satisfechos. Sin embargo, los que tiene una menor satisfacción, con $3.29 \pm 0.92$ y $57.89 \%$ (44), son los de LPCE. En relación con el género, los hombres tienen un promedio $3.60 \pm 0.76$ y un porcentaje de satisfacción de $58.39 \%$. 


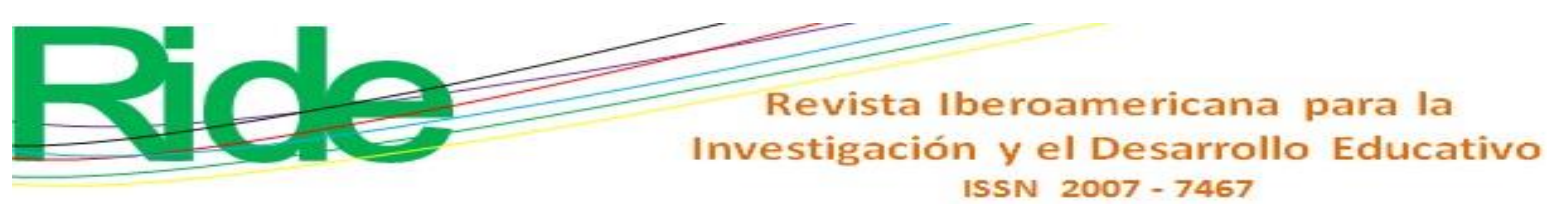

Tabla 2. Grado de satisfacción de la calidad educativa de la carrera de TSUPD y LPCE

\begin{tabular}{|c|c|c|c|c|c|c|c|c|c|}
\hline \multirow{2}{*}{ Variable } & \multicolumn{4}{|c|}{ General } & \multicolumn{2}{|c|}{$<3.5$} & \multicolumn{2}{|c|}{$>3.5$} & \multirow[b]{2}{*}{$\mathbf{p}^{x}$} \\
\hline & $\mathbf{N}$ & Media & DE & $\mathbf{P}$ & $\mathbf{N}$ & $\%$ & $\mathbf{N}$ & $\%$ & \\
\hline Todos & 320 & 3.53 & 0.74 & - & 150 & 46.88 & 170 & 53.12 & - \\
\hline \multicolumn{10}{|c|}{ Grado } \\
\hline LPCE & 76 & 3.29 & 0.92 & & 44 & 57.89 & 32 & 42.11 & \multirow{3}{*}{0.22} \\
\hline TSUPE & 196 & 3.46 & 0.85 & & 97 & 49.49 & 99 & 50.51 & \\
\hline TSUPD & 48 & $4.16^{\mathrm{a}}$ & 0.84 & $<0.001^{*}$ & 9 & 18.75 & 39 & 81.25 & \\
\hline \multicolumn{10}{|c|}{ Género } \\
\hline Hombre & 161 & 3.6 & 0.76 & \multirow{2}{*}{$0.096^{\phi}$} & 67 & 41.61 & 94 & 58.39 & \multirow{2}{*}{0.20} \\
\hline Mujer & 159 & 3.45 & 0.71 & & 83 & 52.2 & 76 & 47.8 & \\
\hline
\end{tabular}

A la izquierda se muestran los promedios generales por grupo $p<0.05$ (Anova, prueba Kruskal Wallis, método de Dunn $\left[{ }^{*}\right], \mathrm{t}$ de Student para muestras independientes $\left.\left.{ }^{\dagger}\right]\right)$. A la derecha, los porcentajes de satisfacción por grupo $p<0.05$ (ji al cuadrado $\left[{ }^{x}\right]$ a grupo que mostró diferencia significativa)

Fuente: Elaboración propia

En la tabla 3 se observa de manera general que los escolares se mostraron satisfecho con los indicadores de Capacitación y habilidad para la enseñanza de los docentes (3.8 \pm 0.86), y sus componentes: Promueven la integración de equipos (3.98 \pm 0.98) y Los docentes tienen la capacidad de comunicación $(3.62 \pm 1.01)$. Seguido por Nivel de autorrealización del estudiante $(3.64 \pm 0.83)$, con los factores: Se fomentan los valores durante tu proceso de enseñanza $(3.74 \pm 0.99)$ y Tus calificaciones las consideras $(3.56 \pm$ 0.96). Y por último, el de Métodos de enseñanza y evaluación (3.62 \pm 0.95$)$, con los elementos: Durante el proceso de enseñanza se incorporan las nuevas tecnologías (3.70 \pm

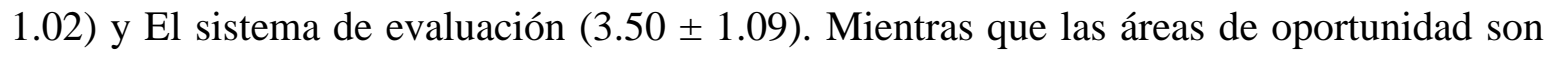
los indicadores Servicios administrativos $(3.41 \pm 1.07)$ y Servicios de apoyo $(3.04 \pm 0.95)$. Este último fue el indicador con el puntaje más bajo en todos los grupos. 


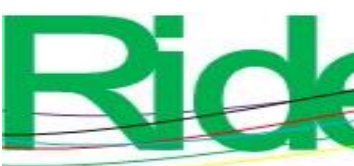

Revista Iberoamericana para la Investigación y el Desarrollo Educativo ISSN 2007 - 7467

Tabla 3. Promedio del grado de satisfacción de la calidad educativa de los estudiantes de

TSUPE y TSUPD, y su continuidad con la LPCE

\begin{tabular}{|c|c|c|c|c|}
\hline INDICADOR/COMPONENTE & LPCE & TSUPD & TSUPE & General \\
\hline \multicolumn{5}{|c|}{ PLAN DE ESTUDIO } \\
\hline $\begin{array}{l}\text { El plan de estudios es pertinente } \\
\text { (necesario, fundamental y relevante) }\end{array}$ & $3.22 \pm 1.01$ & $4.31 \pm 0.85$ & $3.40 \pm 1.03$ & $3.50 \pm 1.06$ \\
\hline $\begin{array}{l}\text { Satisfacción del contenido de las } \\
\text { asignaturas }\end{array}$ & $3.21 \pm 0.98$ & $4.31 \pm 0.80$ & $3.30 \pm 0.95$ & $3.43 \pm 1.01$ \\
\hline $\begin{array}{l}\text { En cuanto a la relevancia de la } \\
\text { información y posibilidad de su } \\
\text { aplicación }\end{array}$ & $3.59 \pm 0.93$ & $4.27 \pm 0.94$ & $3.39 \pm 0.88$ & $3.57 \pm 0.95$ \\
\hline Promedio & $3.34 \pm 0.87$ & $4.30 \pm 0.78^{8}$ & $3.37 \pm 0.85$ & $3.50 \pm 0.91$ \\
\hline \multicolumn{5}{|c|}{ CAPACITACIÓN Y HABILIDAD PARA LA ENSEÑANZA DE LOS DOCENTES } \\
\hline Tienen capacidad de comunicación & $3.24 \pm 1.00$ & $4.40 \pm 0.92$ & $3.58 \pm 0.94$ & $3.62 \pm 1.01$ \\
\hline Conocen y dominan los temas & $3.30 \pm 0.98$ & $4.38 \pm 0.87$ & $3.78 \pm 0.96$ & $3.75 \pm 1.00$ \\
\hline Orientan y apoyan al estudiante & $3.28 \pm 1.07$ & $4.42 \pm 0.96$ & $3.77 \pm 1.01$ & $3.75 \pm 1.07$ \\
\hline El nivel de exigencia de los docentes & $3.39 \pm 1.05$ & $4.48 \pm 0.95$ & $3.80 \pm 1.01$ & $3.81 \pm 1.06$ \\
\hline $\begin{array}{l}\text { Promueven la participación del } \\
\text { estudiante }\end{array}$ & $3.41 \pm 1.02$ & $4.46 \pm 0.87$ & $3.81 \pm 1.05$ & $3.81 \pm 1.06$ \\
\hline Se capacitan y actualizan & $3.36 \pm 1.04$ & $4.50 \pm 0.68$ & $3.84 \pm 0.98$ & $3.82 \pm 1.02$ \\
\hline La responsabilidad del docente & $3.43 \pm 1.05$ & $4.56 \pm 0.82$ & $3.89 \pm 0.92$ & $3.88 \pm 1.00$ \\
\hline Promueven la integración de equipos & $3.62 \pm 0.98$ & $4.48 \pm 0.80$ & $4.00 \pm 0.97$ & $3.98 \pm 0.98$ \\
\hline Promedio & $3.38 \pm 0.84$ & $4.46 \pm 0.75^{\S}$ & $3.81 \pm 0.78$ & $3.8 \pm 0.86$ \\
\hline \multicolumn{5}{|c|}{ MÉTODOS DE ENSEÑANZA Y EVALUACIÓN } \\
\hline Los métodos de enseñanza aplicados & $3.25 \pm 1.01$ & $4.31 \pm 0.80$ & $3.63 \pm 0.90$ & $3.64 \pm 0.97$ \\
\hline $\begin{array}{l}\text { Durante el proceso de enseñanza se } \\
\text { incorporan las nuevas tecnologías }\end{array}$ & $3.42 \pm 1.10$ & $4.40 \pm 0.84$ & $3.64 \pm 0.96$ & $3.70 \pm 1.02$ \\
\hline El sistema de evaluación & $3.28 \pm 1.07$ & $4.27 \pm 0.96$ & $3.40 \pm 1.05$ & $3.50 \pm 1.09$ \\
\hline Promedio & $3.32 \pm 0.95$ & $4.33 \pm 0.77^{\S}$ & $3.56 \pm 0.84$ & $3.62 \pm 0.95$ \\
\hline \multicolumn{5}{|c|}{ NIVEL DE AUTORREALIZACIÓN DEL ESTUDIANTE } \\
\hline Tus calificaciones las consideras & $3.51 \pm 0.81$ & $4.52 \pm 0.71$ & $\mathbf{3 . 3 4} \pm \mathbf{0 . 9 3}$ & $3.56 \pm 0.96$ \\
\hline $\begin{array}{l}\text { Los conocimientos y habilidades } \\
\text { adquiridas durante tu proceso de } \\
\text { enseñanza }\end{array}$ & $3.46 \pm 0.97$ & $4.29 \pm 0.85$ & $3.51 \pm 0.84$ & $3.62 \pm 0.92$ \\
\hline $\begin{array}{l}\text { Capacitación para la inserción al mundo } \\
\text { laboral }\end{array}$ & $3.49 \pm 0.86$ & $4.46 \pm 0.77$ & $3.53 \pm 0.78$ & $3.66 \pm 0.86$ \\
\hline $\begin{array}{l}\text { Se fomentan los valores durante tu } \\
\text { proceso de enseñanza }\end{array}$ & $3.41 \pm 1.05$ & $4.48 \pm 0.80$ & $3.69 \pm 0.93$ & $3.74 \pm 0.99$ \\
\hline Promedio & $3.47 \pm 0.80$ & $4.44 \pm 0.71^{\S}$ & $3.52 \pm 0.75$ & $3.64 \pm 0.83$ \\
\hline \multicolumn{5}{|c|}{ SERVICIOS DE APOYO } \\
\hline $\begin{array}{l}\text { Consideras que el servicio de la } \\
\text { biblioteca }\end{array}$ & $3.39 \pm 1.29$ & $4.13 \pm 1.08$ & $3.44 \pm 1.23$ & $3.53 \pm 1.25$ \\
\hline El acceso a sala de cómputo & $2.87 \pm 1.30$ & $4.21 \pm 1.05$ & $3.63 \pm 1.16$ & $3.54 \pm 1.25$ \\
\hline El acceso a internet & $2.18 \pm 1.31$ & $2.52 \pm 1.47$ & $2.41 \pm 1.30$ & $2.38 \pm 1.33$ \\
\hline
\end{tabular}




\begin{tabular}{|c|c|c|c|c|}
\hline INDICADOR/COMPONENTE & LPCE & TSUPD & TSUPE & General \\
\hline El servicio de fotocopiado & $2.38 \pm 1.28$ & $2.83 \pm 1.42$ & $2.56 \pm 1.25$ & $2.56 \pm 1.28$ \\
\hline El servicio médico & $2.71 \pm 1.25$ & $3.23 \pm 1.43$ & $2.81 \pm 1.30$ & $2.85 \pm 1.32$ \\
\hline $\begin{array}{l}\text { El acceso a actividades culturales, } \\
\text { artísticas y recreativas }\end{array}$ & $3.17 \pm 1.18$ & $3.56 \pm 1.34$ & $3.34 \pm 1.19$ & $3.33 \pm 1.21$ \\
\hline Cafetería & $2.89 \pm 1.01$ & $3.48 \pm 1.03$ & $3.11 \pm 0.94$ & $3.11 \pm 0.99$ \\
\hline Promedio & $2.80 \pm 0.93^{\phi}$ & $3.42 \pm 1.01$ & $3.04 \pm 0.91$ & $3.04 \pm 0.95$ \\
\hline \multicolumn{5}{|c|}{ SERVICIOS ADMINISTRATIVOS } \\
\hline El servicio de control escolar & $3.04 \pm 1.25$ & $3.90 \pm 1.22$ & $3.15 \pm 1.12$ & $3.23 \pm 1.20$ \\
\hline El proceso de admisión e inscripción & $3.53 \pm 1.21$ & $4.10 \pm 1.22$ & $3.48 \pm 1.07$ & $3.58 \pm 1.15$ \\
\hline Promedio & $3.28 \pm 1.11$ & $4.00 \pm 1.12^{\S}$ & $3.31 \pm 0.99$ & $3.41 \pm 1.07$ \\
\hline \multicolumn{5}{|c|}{ AMBIENTE PROPICIO } \\
\hline $\begin{array}{l}\text { La atención por parte del personal } \\
\text { administrativo }\end{array}$ & $3.04 \pm 1.29$ & $3.88 \pm 1.16$ & $3.36 \pm 1.11$ & $3.36 \pm 1.19$ \\
\hline $\begin{array}{l}\text { La atención del personal de servicios de } \\
\text { apoyo y mantenimiento }\end{array}$ & $3.43 \pm 1.19$ & $3.94 \pm 1.16$ & $3.65 \pm 1.00$ & $3.64 \pm 1.08$ \\
\hline $\begin{array}{l}\text { La atención por parte del personal } \\
\text { docente }\end{array}$ & $3.38 \pm 1.13$ & $4.44 \pm 0.74$ & $3.70 \pm 1.06$ & $3.73 \pm 1.08$ \\
\hline El ambiente estudiantil & $3.41 \pm 1.09$ & $4.46 \pm 0.87$ & $3.52 \pm 1.10$ & $3.63 \pm 1.12$ \\
\hline Promedio & $3.32 \pm 0.93$ & $4.18 \pm 0.72^{\S}$ & $3.56 \pm 0.88$ & $3.59 \pm 0.90$ \\
\hline \multicolumn{5}{|c|}{ INFRAESTRUCTURA } \\
\hline Limpieza de las instalaciones & $3.47 \pm 1.19$ & $3.83 \pm 1.23$ & $3.78 \pm 1.12$ & $3.71 \pm 1.16$ \\
\hline Condiciones del mobiliario & $3.33 \pm 1.12$ & $4.08 \pm 1.18$ & $3.56 \pm 1.00$ & $3.58 \pm 1.08$ \\
\hline $\begin{array}{l}\text { Espacios para la enseñanza (salones, } \\
\text { talleres, laboratorios) }\end{array}$ & $3.58 \pm 1.10$ & $4.38 \pm 0.89$ & $3.70 \pm 0.97$ & $3.77 \pm 1.02$ \\
\hline Espacios para el descanso y recreación & $3.18 \pm 1.26$ & $4.23 \pm 1.10$ & $3.42 \pm 1.09$ & $3.49 \pm 1.18$ \\
\hline Instalaciones de la biblioteca & $3.51 \pm 1.17$ & $4.17 \pm 1.23$ & $3.54 \pm 1.10$ & $3.63 \pm 1.16$ \\
\hline Instalaciones del área de cómputo & $3.22 \pm 1.15$ & $4.33 \pm 0.97$ & $3.56 \pm 1.08$ & $3.60 \pm 1.13$ \\
\hline Instalaciones de la cafetería escolar & $3.47 \pm 1.18$ & $4.21 \pm 0.97$ & $3.35 \pm 1.13$ & $3.51 \pm 1.16$ \\
\hline Instalaciones deportivas & $3.46 \pm 1.05$ & $4.00 \pm 1.11$ & $3.44 \pm 1.12$ & $3.53 \pm 1.12$ \\
\hline Instalaciones sanitarias & $3.41 \pm 1.11$ & $3.90 \pm 1.12$ & $3.38 \pm 1.05$ & $3.46 \pm 1.09$ \\
\hline Promedio & $3.40 \pm 0.91$ & $4.13 \pm 0.84^{\S}$ & $3.52 \pm 0.83$ & $3.59 \pm 0.88$ \\
\hline \multicolumn{5}{|c|}{ PROMEDIO GENERAL } \\
\hline & $3.27 \pm 0.74$ & $4.13 \pm 0.64^{\S}$ & $3.48 \pm 0.69$ & $3.53 \pm 0.74$ \\
\hline
\end{tabular}

Negritas: puntuación más alta; negritas cursivas: puntuación baja. Los datos se expresan en media, DE. ${ }^{\S} p<0.001 ;{ }^{\phi} \mathrm{p}<$ 0.02 (Kruskal-Wallis, Anova, Método de Dunn).

Fuente: Elaboración propia

En la figura 2 se muestra el porcentaje de satisfacción (> 3.5) de cada uno de los indicadores. De manera general, $53.13 \%$ (170) se encuentra satisfecho, con $67.81 \%$ (217) en el rubro La capacitación y habilidad para la enseñanza de los docentes, seguido de Ambiente propicio y de Nivel de autorrealización con 62.81 \% (201) y $60.62 \%$ (194) respectivamente. El porcentaje más bajo se encontró en Servicios de apoyo, con 38.12 \% 


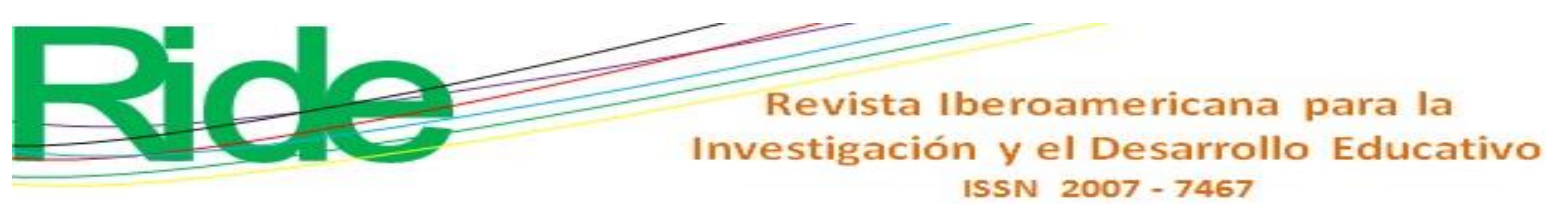

(122). Los alumnos de TSUPD presentaron una satisfacción de $81.25 \%$ (39), con un rango de $85.41 \%$ (41) a $47.91 \%$ (23), mientras que los de LPCE expresaron $42 \%$ (32), y un rango de $56.58 \%$ (43) a $34.21 \%$ (26).

Figura. 2. Porcentaje de satisfacción de la calidad educativa de los estudiantes encuestados

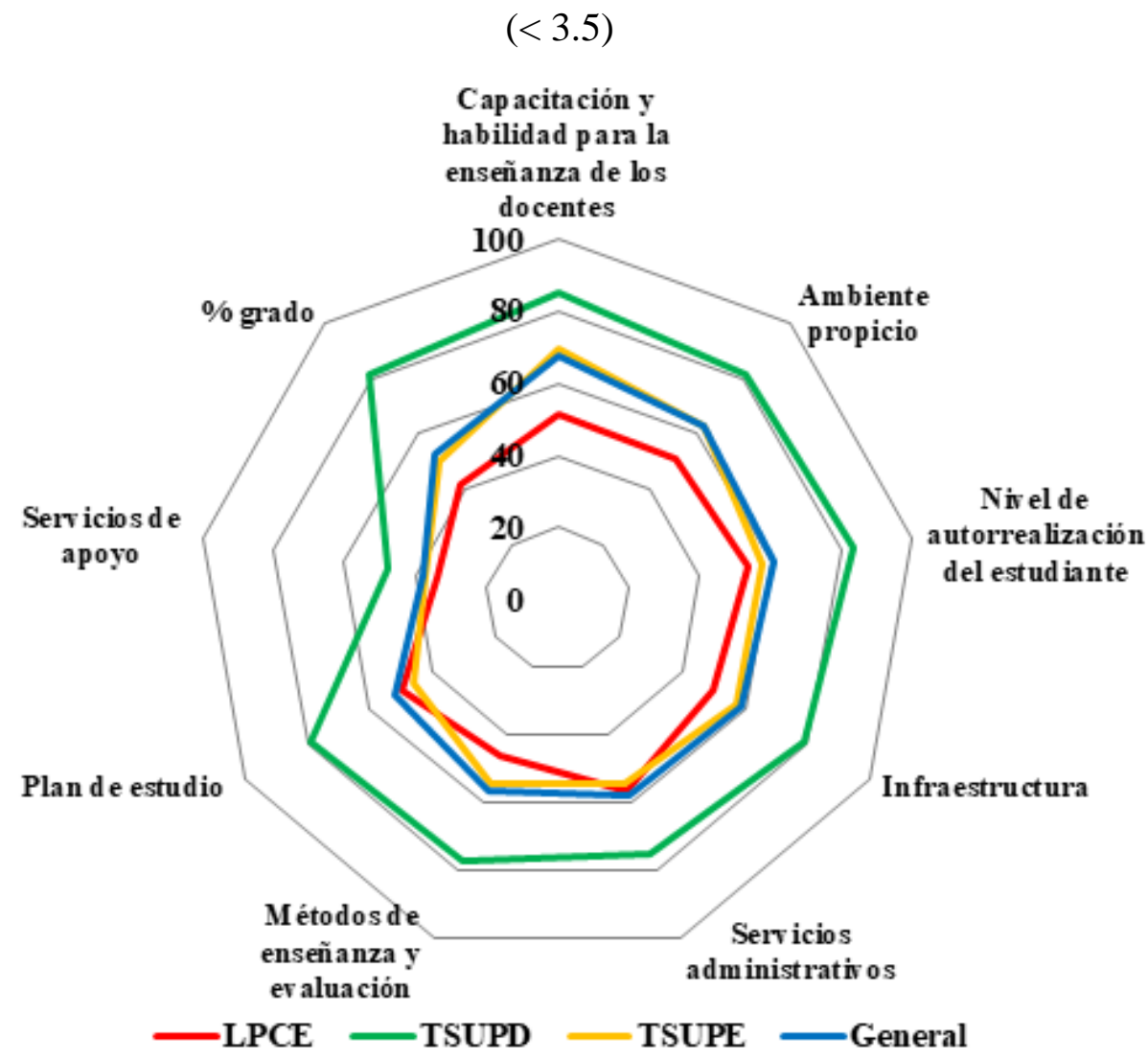

Fuente: Elaboración propia

\section{Análisis de correlación de Pearson}

Para la construcción de la tabla 4 solo se consideraron las correlaciones $\geq 0.8$ ( $<$ 0.001). La capacitación para la inserción al mundo laboral y Los conocimientos y habilidades adquiridas durante el proceso de enseñanza, ambos del indicador Nivel de autorrealización, correlacionaron muy fuerte en todos los grupos $(\geq 0.8)$. En el indicador Capacitación y habilidad para la enseñanza, Los docentes promueven la participación del estudiante y Los docentes orientan y apoyan al estudiante, tanto en el caso de los estudiantes de licenciatura como en de los del despresurizado, tienen un valor superior a 


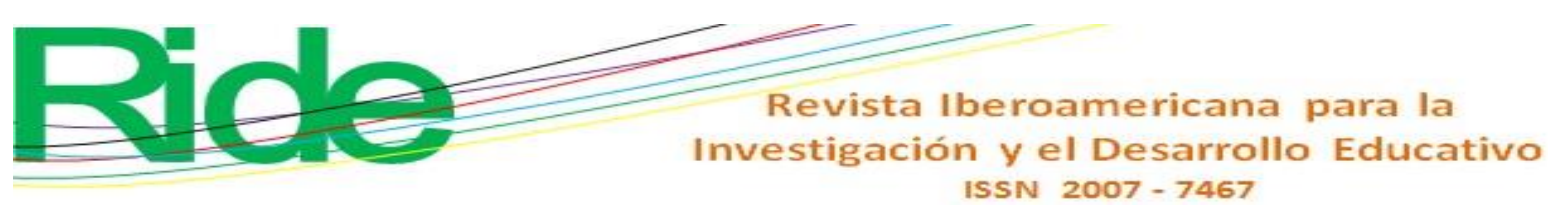

0.8. El grupo de TSUPD fue el que presentó un número mayor de correlaciones, algunas con valores arriba de 0.9 .

Tabla 4. Resumen de la correlación de Pearson de los diferentes indicadores de la calidad educativa de los estudiantes de TSUPE y despresurizada (TSUPD), y LPCE

\begin{tabular}{|c|c|c|c|c|c|c|}
\hline 苍 & \multicolumn{2}{|c|}{ COMPONENTE } & \multirow{2}{*}{$\begin{array}{c}\text { LPCE } \\
\\
0.727\end{array}$} & \multirow{2}{*}{$\begin{array}{c}\text { TSUPE } \\
\\
0.647\end{array}$} & \multirow{2}{*}{$\begin{array}{c}\text { TSUPD } \\
0.847\end{array}$} & \multirow{2}{*}{$\begin{array}{c}\text { General } \\
\\
0.7\end{array}$} \\
\hline 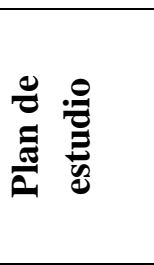 & $\begin{array}{l}\text { Tu grado de } \\
\text { satisfacción en } \\
\text { cuanto al } \\
\text { contenido de las } \\
\text { asignaturas }\end{array}$ & $\begin{array}{l}\text { Consideras que el plan de } \\
\text { estudios es pertinente } \\
\text { (necesario, fundamental y } \\
\text { relevante) }\end{array}$ & & & & \\
\hline \multirow{7}{*}{ 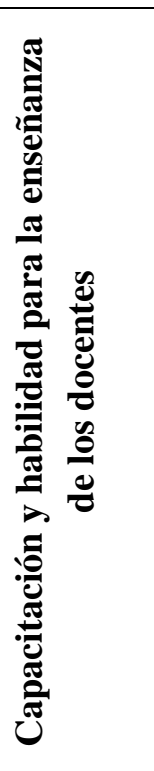 } & \multirow{2}{*}{$\begin{array}{l}\text { Los docentes } \\
\text { conocen y } \\
\text { dominan los } \\
\text { temas }\end{array}$} & $\begin{array}{l}\text { Los docentes se capacitan y } \\
\text { actualizan }\end{array}$ & 0.807 & 0.758 & 0.718 & 0.791 \\
\hline & & $\begin{array}{l}\text { Los docentes tienen } \\
\text { capacidad de comunicación }\end{array}$ & 0.752 & 0.644 & 0.854 & 0.73 \\
\hline & \multirow{3}{*}{$\begin{array}{l}\text { Los docentes } \\
\text { promueven la } \\
\text { participación del } \\
\text { estudiante }\end{array}$} & $\begin{array}{l}\text { La responsabilidad del } \\
\text { docente }\end{array}$ & 0.777 & 0.663 & 0.817 & 0.738 \\
\hline & & $\begin{array}{l}\text { Los docentes orientan y } \\
\text { apoyan al estudiante }\end{array}$ & 0.801 & 0.649 & 0.905 & 0.744 \\
\hline & & $\begin{array}{l}\text { Los docentes promueven la } \\
\text { integración de equipos }\end{array}$ & 0.69 & 0.716 & 0.928 & 0.753 \\
\hline & \multirow{2}{*}{$\begin{array}{l}\text { Los docentes } \\
\text { orientan y } \\
\text { apoyan al } \\
\text { estudiante }\end{array}$} & $\begin{array}{l}\text { La responsabilidad del } \\
\text { docente }\end{array}$ & 0.761 & 0.636 & 0.906 & 0.736 \\
\hline & & $\begin{array}{l}\text { La atención por parte del } \\
\text { personal docente }\end{array}$ & 0.807 & 0.558 & 0.752 & 0.675 \\
\hline \multirow{2}{*}{ 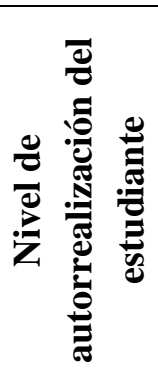 } & \multirow{2}{*}{$\begin{array}{l}\text { Capacitación } \\
\text { para la inserción } \\
\text { al mundo } \\
\text { laboral }\end{array}$} & $\begin{array}{l}\text { Los conocimientos y } \\
\text { habilidades adquiridas } \\
\text { durante tu proceso de } \\
\text { enseñanza }\end{array}$ & 0.848 & 0.856 & 0.864 & 0.869 \\
\hline & & $\begin{array}{l}\text { Se fomentan los valores } \\
\text { durante tu proceso de } \\
\text { enseñanza }\end{array}$ & 0.771 & 0.788 & 0.915 & 0.817 \\
\hline 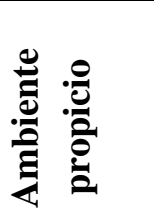 & $\begin{array}{l}\text { La atención por } \\
\text { parte del } \\
\text { personal } \\
\text { administrativo }\end{array}$ & $\begin{array}{l}\text { El proceso de admisión e } \\
\text { inscripción }\end{array}$ & 0.689 & 0.698 & 0.878 & 0.727 \\
\hline
\end{tabular}




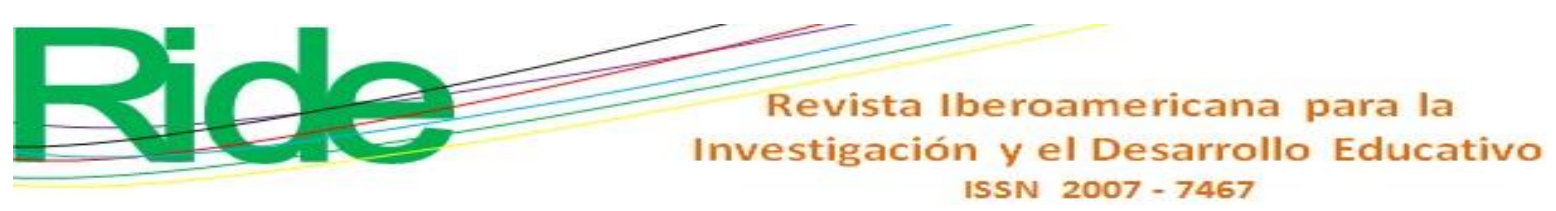

Solo se muestran aquellos con un valor $\geq 0.8(p<0.001)$. En cursiva las puntuaciones más altas

Fuente: Elaboración propia

\section{Análisis de principales componentes}

La medida de la adecuación muestral de KMO fue de 0.95, por lo que se procedió a realizar el análisis por componentes principales: se obtuvieron de cinco a ocho componentes que explican un rango de $66 \%$ a $81 \%$ de la varianza. En la tabla 5 se muestran los cinco ítems, con un puntaje mayor en el primer componente. El grupo de TSUPD fue el que mostró ítems puntuados con 0.874 y 0.8 ; son los únicos que consideran el plan de estudios pertinente (0.849). Destaca que los estudiantes en todos los grupos consideran como importante en la calidad educativa el factor Atención por parte del docente, puntuado entre 0.858 y 0.811 (negritas). Seguido de Se fomentan los valores durante tu enseñanza (cursivas) y Los docentes promueven la participación de los estudiantes (cursivas). 


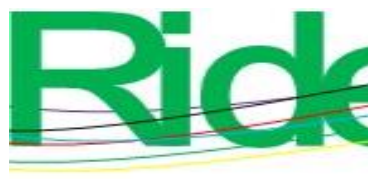
Investigación y el Desarrollo Educativo ISSN $2007-7467$

\section{Discusión}

La profesionalización en el área de la salud no es nueva. Comenzando con la medicina, se estableció desde hace siglos. En décadas recientes, esta tendencia se aceleró y la expectativa actual es que todos los participantes de esta área adquieran una educación universitaria, como ha sucedido con la fisioterapia (década de los 70) y la enfermería (década de los 80). Los primeros intentos de programas de entrenamiento en atención prehospitalaria en Latinoamérica comenzaron en 1979 aunque sin mucho éxito (Devenish, 2014; Kennedy, Kenny y O’Meara, 2015).

La legislación de la atención médica prehospitalaria en México se inició en 1987, después del terremoto del 85 (al igual que el Sistema Nacional de Protección Civil), con la Norma Técnica 358 para la Prestación de Servicios de la Atención Médica de las Unidades Terrestres de Urgencias y Cuidados Intensivo. A lo que le han seguido diversas normas: la NOM 020-SSA-1994 para la Prestación de Servicios de Atención Médica en Unidades Móviles Tipo Ambulancia (2000); posteriormente la NOM-237-SSA1-2004 Regulación de los Servicios de Salud, Atención Prehospitalaria de las Urgencias (2006), hasta su última modificación, en 2012, con la NOM-034-SSA3-2013 Regulación de los Servicios de Salud, Atención Médica Prehospitalaria. En 2007, en el artículo 79 de la Ley General de Salud, surge la figura del técnico en atención médica prehospitalaria, la cual se reforma en el 2015, cuando se le comienza a demandar un diploma avalado por una institución educativa competente (Secretaría de Salud/Secretariado Técnico del Consejo Nacional para la Prevención de Accidentes [STCONAPRA], 2017).

El personal de atención prehospitalaria, dentro del sector de atención médica de emergencia, tiene la finalidad de reducir la incidencia de discapacidad a corto plazo y mejorar la calidad de vida al disminuir notablemente las consecuencias y la morbimortalidad, por lo que los paramédicos están encargados de la evaluación, el tratamiento y el transporte seguro de los pacientes al hospital (Devenish, 2014; Kennedy et al., 2015).

Con esta finalidad la UTVT oferta desde hace 10 años el TSUP y su continuación con la LPCE (2012). Esta institución pertenece al Subsistema de las Universidades Tecnológicas, con categoría 5B, y cuenta, además, con programas educativos de corta duración (dos años, seis cuatrimestres en la opción Técnico Superior Universitario), basados en competencias con $70 \%$ de práctica y $30 \%$ de teoría, con el objeto de 


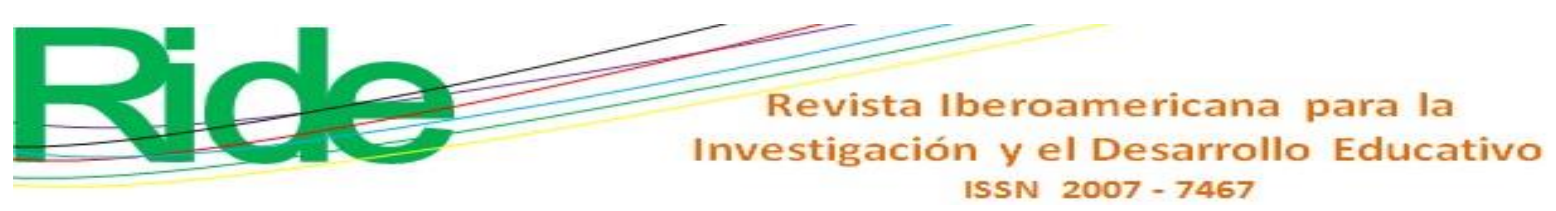

proporcionar al estudiante una rápida inserción al mercado laboral (Rama, Claudio., 2015; Ruiz Larraguivel, 2014). Una de las limitaciones de este estudio fue la falta de participación por parte de los estudiantes (22\%); solo participó $78 \%$ de la matrícula, distribuidos por nivel académico. El mayor porcentaje se observa en los TSUPE, con $61 \%$, mientras que la LPCE tiene $24 \%$ y TSUPD $15 \%$. Esto es debido a que, en estos dos últimos, el número de estudiantes y grupos es menor, además de que no todos contestaron la encuesta.

Al ser la atención al cliente una de sus dimensiones fundamentales para la calidad, su satisfacción es trascendental, por lo que las organizaciones deben comprender sus necesidades, satisfacer sus requisitos y esforzarse por exceder sus expectativas (Agustín y Domelis, 2009; Cadena, Mejías, Vega y Vásquez, 2015). En el caso de las universidades, donde los estudiantes son los destinatarios de los esfuerzos universitarios, su satisfacción es uno de los indicadores de calidad más importantes. Sin embargo, no es un hecho observable, es una aproximación a la percepción que tienen los alumnos acerca de sus estudios, que revela cómo se está generando el proceso de enseñanza-aprendizaje, por lo que se ha convertido en un aspecto estratégico que marca la diferencia en el sistema universitario actual. Con el tiempo podría considerarse como una medida de control de calidad de dicho proceso (Cevallos, 2014; Oliva y Gómez, 2014; Pérez y Pereyra, 2015; Surdez, Sandoval y Lamoyi, 2018).

Para esta investigación se utilizó el instrumento para evaluar la satisfacción del estudiante de Álvarez et al. (2015) debido a que tiene un sustento bibliográfico amplio, además de contar con análisis de confiabilidad, por lo que se consideró pertinente. Con base en una escala Likert ( 1 = Muy insatisfecho, 5 = Totalmente satisfecho), califica diversos aspectos, como es el plan de estudios, la habilidad docente, los métodos de enseñanzaevaluación, la infraestructura, entre otros.

Aquí, $35 \%$ de los estudiantes eligieron la opción "Muy satisfechos" (4) y $26.3 \%$ "Satisfechos" (3), lo que explica el promedio obtenido de 3.5 (tabla 2). Por lo anterior, los alumnos se encuentran entre satisfechos y muy satisfechos. Lo anterior también denota que consideran una calidad positiva, por lo que se utilizó como punto de corte para valorar la satisfacción en la calidad educativa, la cual, cabe señalar, es ligeramente superior a la reportada por los propios Álvarez et al. (2015). Los indicadores que aparecen con mayor frecuencia en el promedio y el porcentaje de satisfacción altos son los relacionados a la capacitación y la habilidad para la enseñanza de los docentes y el nivel de autorrealización. 


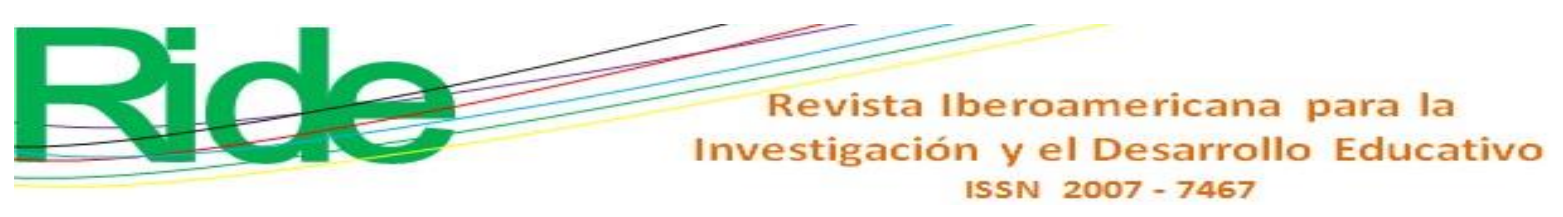

Mientras que los vinculados con los servicios de apoyo se ubican en el último lugar en todos los grupos.

Respecto al grado con mayor satisfacción, los alumnos de TSUPD obtuvieron un promedio de $4.13 \pm 0.64$, que es significativo al compararlos con los otros grupos ( $p<$ 0.001 ), con $81.25 \%$ de satisfechos, siendo La capacitación y habilidad para la enseñanza de los docentes y Nivel de autorrealización los indicadores con puntajes y porcentajes más altos. Esto podría ser explicado porque la mayoría acuden con la convicción de profesionalizarse, por lo que el contenido temático de las asignaturas les aporta conocimiento científico y tecnológico, que mejora su práctica en su trabajo.

Sin embargo, el promedio de todos los indicadores en los de LPCE se encuentran por debajo del punto de corte $\left(42 \%\right.$, y $\left.3.27 \pm 0.74^{2}\right)$, por lo que tienen una menor satisfacción. Los indicadores Plan de estudio (46.05 \% y $3.34 \pm 0.87)$ y Métodos de enseñanza y evaluación (50 \% y $3.32 \pm 0.95)$ se encuentran por arriba del de Servicios de apoyo $(34.21 \%$ y $2.80 \pm 0.93, \mathrm{p}<0.02)$. Este último es el promedio más bajo de todos (ver tabla 3 y figura. 2).

Una de las causas podría ser la falta de conocimiento por parte de los alumnos del contenido de las materias de la licenciatura. Aunque pudieran considerarse que ambas fungen como áreas operativas, cada una representa un esquema diverso de atención. El TSUP es técnico operativo y con respuesta directa a la población; mientras que el área de Protección Civil se encarga del manejo integral del riesgo en la sociedad, para la reducción, mitigación y control de los agentes perturbadores. Además de la escasez de profesores con experiencia en el área que deseen dar clases, debido a múltiples factores como el horario vespertino, la ubicación de la universidad y la remuneración económica, aunado a que hasta antes del 2012 no existía.

Como sucede en otras áreas, la presencia de la mujer en la universidad se ha incrementado en las últimas tres décadas (Navarro y Casero, 2012), fenómeno que se observa en la distribución 1:1; por lo que hay un porcentaje menor de varones en los de LPCE, con $42.1 \%$, a diferencia de los TSUPD, con $75 \%$, debido que la mayoría de los paramédicos en activo son hombres. Está reportado que el género es uno de los factores que mayor influencia ejerce en la elección de la carrera universitaria, por ejemplo, el femenino se asocia más a las ciencias de la salud y sociales (Martínez, Castro, Zurita y Lucena,

\footnotetext{
${ }^{2}$ Porcentaje de satisfacción y promedio en caso de un indicador, y promedio si se trata de un componente.
} 


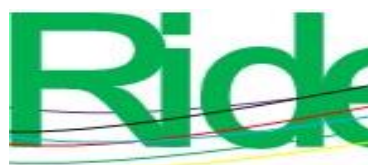

Revista Iberoamericana para la
Investigación y el Desarrollo Educativo
ISSN $2007-7467$

2015). Sin embargo, hay estudios que muestran que esta suposición es empírica porque han existido errores de medición, o bien porque únicamente reflejan diferencias a largo de la historia de la socialización (Rodríguez, Sánchez y Labajos, 2017).

En relación precisamente con el género, $58 \%$ de los varones se encuentra satisfecho con la educación que reciben, con un promedio $3.6 \pm 0.76$, mientras que es menor el porcentaje y el promedio en las mujeres, sin haber diferencias significativas. No está de más señalar que también existen trabajos que indican que las mujeres dedican más esfuerzo y tiempo a estudiar que los hombres, y por lo tanto son más exigentes (Martínez et al., 2015).

Ahora bien, $50.94 \%$ (163) de los encuestados se dedican a estudiar; el grupo de TSUPE registra este rasgo con el mayor porcentaje: $62.76 \%$ (123). Y $24.69 \%$ (79) laboran en atención prehospitalaria o área afín, con $93.75 \%$ (45) los TSUPD, pues uno de los requisitos para poder ingresar a esta modalidad es estar trabajando como paramédicos en alguna institución pública o privada, con una antigüedad de por lo menos cinco años.

Siguiendo a Álvarez et al. (2015), la calidad es un elemento clave para que una organización sea competitiva. Los estudiantes no solo ingresan a las universidades por la obtención de un título, sino que se ven influenciados por la calidad de los conocimientos y la educación docente (Inzunza et al., 2015). Se han identificado numerosos elementos que afectan el grado de satisfacción de los alumnos, que influyen en el rendimiento académico, la deserción o el sentimiento de pertenencia. Algunos de ellos son los siguientes: los docentes, las oportunidades de la carrera, las instalaciones, la reputación de la universidad, entre otros (Surdez et al., 2018).

En el análisis de correlación se observó que para los estudiantes es importante la autorrealización $(60.6 \%$ y $3.64 \pm 0.83)$, viéndose reflejada en la capacitación para la inserción del mundo laboral (3.66 \pm 0.86) con la adquisición de conocimientos y habilidades (3.62 \pm 0.92); por lo que la educación universitaria está obligada a brindar las herramientas necesarias para que los educandos sean críticos e innovadores mediante la generación de conocimiento que permita el desarrollo sustentable de la nación (Espinosa, 2016). Otro aspecto destacable es el fomento de los valores; los estudiantes de TSUPD $(4.48 \pm 0.80)$, al ser activos laboralmente, conocen las implicaciones de una mala praxis o una conducta inadecuada. El modelo basado en competencias que rige el sistema de las universidades tecnológicas desarrolla los valores profesionales, tal y como son la actitud profesional y la ética, a través de las competencias transversales. 


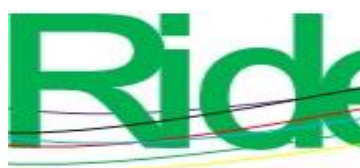

Revista Iberoamericana para la
Investigación y el Desarrollo Educativo
ISSN $2007-7467$

La evaluación del docente por parte de los educandos se ha asumido como una medida directa de la satisfacción con la experiencia universitaria, y de la calidad de la misma (Moreira y Santos, 2016). Este estudio corrobora que la atención por parte del docente $(3.73 \pm 1.08)$, perteneciente aquí al indicador Ambiente propicio (63\% satisfacción y $3.59 \pm 0.9$ de promedio), es un factor determinante para la satisfacción, como lo secunda el APC. Al respecto, existen reportes que sugieren que si los alumnos perciben relaciones positivas con el cuerpo docente son más propensos a experimentar mayor satisfacción y compromiso (Inzunza et al., 2015).

Las características de los docentes que los estudiantes valoran se manifestaron en el indicador Capacitación y habilidad para la enseñanza de los docentes (68 \% y $3.8 \pm 0.86$ ), con los componentes Responsabilidad del docente $(3.88 \pm 1.00)$, Los docentes promueven la participación del estudiante $(3.81 \pm 1.06)$ y Los docentes orientan y apoyan al estudiante $(3.75 \pm 1.07)$, que correlacionan de fuerte a muy fuerte, como lo muestra el análisis de correlación. Sin embargo, la capacidad de comunicación $(3.62 \pm 1.01)$ es el componente con menor promedio, por lo que se le considera un área de oportunidad detectada; junto con Los docentes se capacitan y actualizan (3.75 \pm 1.00$)$, que correlaciona con Los docentes conocen y dominan el tema $(3.82 \pm 1.02)$, de fuertes a muy fuertes.

Aunque la calidad académica y pedagógica de la enseñanza son determinantes cruciales de la satisfacción de los estudiantes, no se deben descuidar otros elementos como lo son la infraestructura $(59.06 \%$, y $3.59 \pm 0.88)$, los servicios administrativos $(58.13 \%$ y $3.41 \pm 1.07)$ y la calidad de los servicios $(38.13 \%$ y $3.04 \pm 0.95)$ (Inzunza et al., 2015). Es necesario contar con instalaciones limpias y modernas, mobiliario cómodo y espacios adecuados al estudio; la literatura apoya una relación positiva entre la calidad del servicio y la satisfacción (Alonso Dos Santos, 2016).

La infraestructura del Laboratorio de Paramédico fue valorada con un promedio de $3.77 \pm 1.02$, por lo que se considera que los estudiantes se encuentran satisfechos. Este cuenta con simuladores médicos, diversos maniquís para la práctica de reanimación cardiopulmonar, brazos para canalización, equipo electromédico como desfibriladores y monitores; recursos que contribuyen a desarrollar las competencias de una buena práctica profesional. En cuanto al indicador Servicios administrativos $(3.41 \pm 1.07)$, Proceso de admisión e inscripción $(3.58 \pm 1.15)$ correlaciona de fuerte a muy fuerte con La atención por parte del personal administrativo $(3.36 \pm 1.19)$ del indicador Ambiente propicio. 


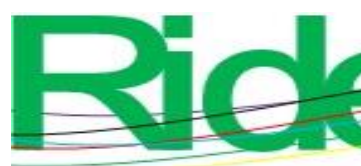

Revista Iberoamericana para la Investigación y el Desarrollo Educativo ISSN $2007-7467$

Cabe destacar que los servicios de apoyo tienen $62 \%$ de insatisfacción y un promedio de $3.04 \pm 0.95$ : es el promedio más bajo de todos los indicadores. Es probable que, debido a la ubicación alejada de las universidades tecnológica, se vean afectados algunos servicios de apoyo, como lo son el acceso a internet $(2.38 \pm 1.33)$, el servicio de fotocopiado $(2.56 \pm 1.28)$, el servicio médico $(2.85 \pm 1.32)$ y la cafetería $(3.11 \pm 0.99)$. En cuanto a este punto no está de más añadir que recientemente se han implementado actividades culturales, artísticas y recreativas, a través de talleres de teatro, fotografía, ajedrez y deportes.

\section{Conclusiones}

Desde hace 10 años se oferta en la UTVT, el TSUP tanto en modalidad escolarizada como despresurizada, con continuidad en la LPCE, esta última con una duración de seis años, con lo que se contribuye al desarrollo de la región.

Si bien los estudios de satisfacción en la calidad académica en los estudiantes son controversiales, al considerar que su opinión puede estar sesgada por el resultado del momento, permiten a las instituciones de educación superior encontrar puntos de mejora continua, pues, en última instancia, son estos, los alumnos, los destinatarios de los procesos de enseñanza.

Los estudiantes de TSUPD son los que se encuentran muy satisfechos con la educación recibida, debido a que la información científica y técnica que reciben complementa su práctica diaria, y mejora su experiencia laboral. Mientras que los de LPCE están solo satisfechos debido a múltiples factores, como lo son el desconocimiento del contenido de la licenciatura o la escasez de profesores.

La atención personal por parte del docente es primordial para una educación con calidad, de acuerdo con la percepción de los estudiantes de la Dirección de Carrera de Paramédico y Protección Civil. Es responsabilidad de este, con su proceso educativo, al promover su participación, y darles orientación y apoyo.

En el indicador Plan de estudio, la mayoría de los grupos expresaron su satisfacción en cuanto al contenido de las asignaturas, y se relaciona con el de Nivel de autorrealización, debido a que los capacita con conocimientos y habilidades para la inserción al mundo laboral, además de fomentar los valores; en consecuencia, los estudiantes de TSUP consideran que el programa es pertinente. 


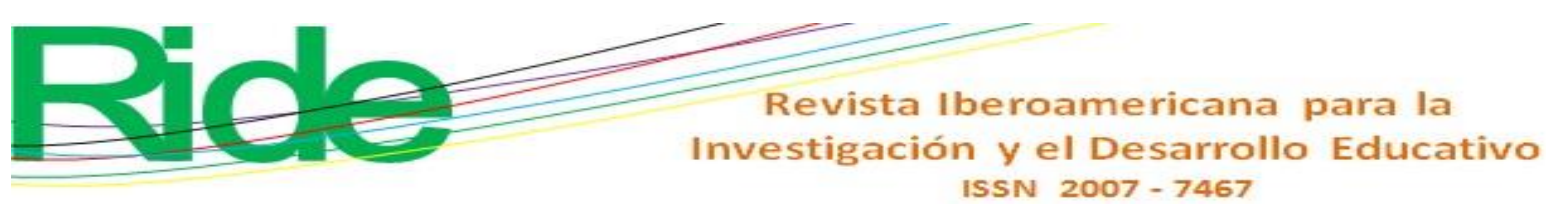

Los servicios de apoyo son un área de oportunidad para la Dirección de Paramédico y Protección Civil, en donde el servicio médico, el de fotocopiado y el acceso a internet tienen los promedios más bajos.

Este primer ejercicio de evaluación permitió identificar el grado de satisfacción de los estudiantes de TSUP y LPCE, el cual es de satisfechos a muy satisfechos, además de conocer su opinión con respecto a lo que consideran una educación de calidad. Para futuras investigaciones sería importante realizar entrevistas personales o grupales con la finalidad de indagar las inquietudes de los estudiantes y atender sus necesidades, así como realizar el cuestionario por lo menos una vez al año de manera rutinaria para construir una cultura de mejora continúa. 


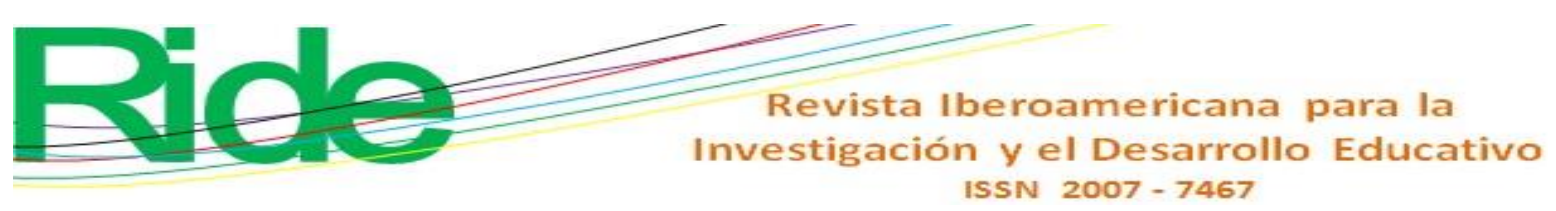

\section{Referencias}

Agustín, M. y Domelis, M. (2009). Desarrollo de un instrumento para medir la satisfacción estudiantil en educación superior. Docencia Universitaria, 10(2), 29-47.

Alonso Dos Santos, M. (2016). Calidad y satisfacción: el caso de la Universidad de Jaén. Revista de la Educación Superior, 45(178), 79-95. Recuperado de https://doi.org/10.1016/j.resu.2016.02.005.

Álvarez, J., Chaparro, E. y Reyes, D. (2015). Estudio de la Satisfacción de los Estudiantes con los Servicios Educativos brindados por Instituciones de Educación Superior del Valle de Toluca. REICE. Revista Iberoamericana sobre Calidad, Eficacia y Cambio en Educación., 13(2), 5-26.

Cadena, M., Mejías, A., Vega, A. y Vásquez, J. (2015). La satisfacción estudiantil universitaria: análisis estratégico a partir del análisis de factores. Industrial Data, 18(1), 9-18.

Cevallos, L. I. (2014). Análisis sobre competencias y satisfacción de los estudiantes de Grado en Administración y Dirección de Empresas de la Universidad Politécnica de Cartagena (Tesis de Licenciatura). Universidad Politécnica de Cartagena, Cartagena, Colombia.

Chang, Y. T., Tsai, K. C. and Williams, B. (2017). What are the educational and curriculum needs for emergency medical technicians in Taiwan? A scoping review. Advances in Medical Education and Practice, Volume 8, 649-667. Recuperado de https://doi.org/10.2147/AMEP.S140839.

Devenish, A. S. (2014). Experiences in becoming a paramedic: a qualitative study examining the professional socialisation of university qualified paramedics. (doctoral dissertation). Queensland University of Technology, Brisbane, Queensland, Australia.

Espinosa, E. M. (2016). RESEÑA. Revista de la Educación Superior, 45(177), 177-182. Recuperado de https://doi.org/10.1016/j.resu.2016.04.006.

González, M., Pino, M. y Penado, M. (2017). Estudio de la satisfacción percibida por los estudiantes de la UNED con su vida universitaria. RIED. Revista Iberoamericana de Educación a Distancia, 20(1), 243. Recuperado de https://doi.org/10.5944/ried.20.1.16377. 


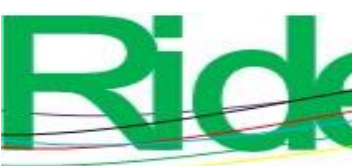

Revista Iberoamericana para la Investigación y el Desarrollo Educativo ISSN $2007-7467$

Hou, X. Y., Rego, J. and Service, M. (2013). Review article: Paramedic education opportunities and challenges in Australia: Paramedic education in Australia. Emergency Medicine Australasia, 25(2), 114-119. Recuperado de https://doi.org/10.1111/1742-6723.12034.

Inzunza, B, Ortiz, L., Pérez, C, Torres, G., McColl, P, Meyer, A., Matus, O., Bastías, N. y Bustamante, C. (2015). Estructura Factorial y Confiabilidad del Cuestionario de Satisfacción Académica en Estudiantes de Medicina Chilenos. Revista Iberoamericana de Diagnóstico y Evaluación - e Avaliação Psicológica, 2(40), 7382. Recuperado de https://www.redalyc.org/pdf/4596/459645432008.pdf.

Kennedy, S., Kenny, A. y O’Meara, P. (2015). Student paramedic experience of transition into the workforce: A scoping review. Nurse Education Today, 35(10), 1037-1043. Recuperado de https://doi.org/10.1016/j.nedt.2015.04.015.

Martínez, A., Castro, M., Zurita, F. and Lucena, M. (2015). La elección de estudios superiores universitarios en función de la modalidad de estudios, la nota media y el género. $\quad$ Magister, 27(1), 18-25. Recuperado de https://doi.org/10.1016/j.magis.2015.06.001.

Moreira, L. M. y Santos, M. Á. (2016). Evaluando la enseñanza en la Educación Superior: percepciones de docentes y discentes. Revista electrónica de investigación educativa, 18(3), 19-36.

Navarro, C. y Casero, A. (2012). Análisis de las diferencias de género en la elección de estudios universitarios. Estudios sobre educación, 2, 115-132.

Oliva, E. J. D. y Gómez, Y. D. (2014). Evolución conceptual de los modelos de medición de la percepción de calidad del servicio: Una mirada desde la educación superior. Suma de negocios, 5(12), 180-191.

Osorio, M. del C. y Parra, L. (2016). La satisfacción escolar en los estudiantes del primer año de la carrera de Médico Cirujano. Investigación en Educación Médica, 5(17), 310. Recuperado de https://doi.org/10.1016/j.riem.2015.08.002.

Pérez, I. y Pereyra, E. (2015). Satisfacción estudiantil: un indicador de la Calidad educativa en el Departamento de Biología Celular, UCV. Revista de Pedagogía, 36(99), 6989.

Rama, C. (2015). La conformación diferenciada de un nuevo subsistema tecnológico universitario en América Latina. Revista de la Educación Superior, 44(173), 11-46. 


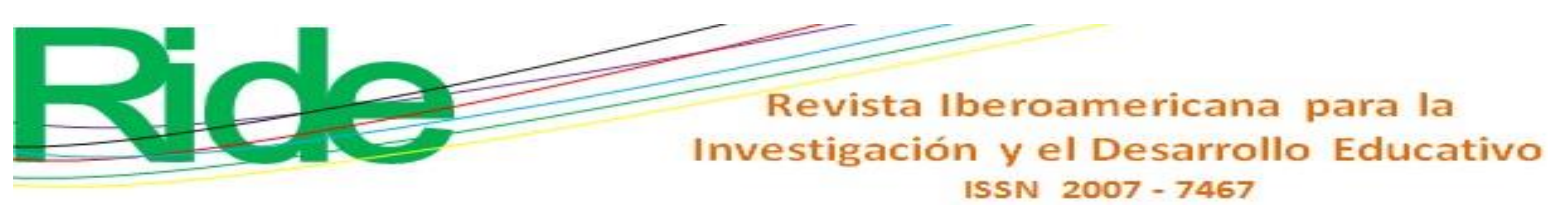

Rodríguez, M. C., Sánchez, E. y Labajos, M. T. (2017). Vocación ocupacional y género en estudiantes universitarios de ciencias de la salud. Revista Latinoamericana de Ciencias Sociales, Niñez y Juventud, 15(1), 345-356.

Ruiz Larraguivel, E. (2014). Las empresas como espacios para el aprendizaje ocupacional. Perfiles Educativos, 36(144), 69-84. Recuperado de https://doi.org/10.1016/S01852698(14)70624-5.

Secretaría de Salud/Secretariado Técnico del Consejo Nacional para la Prevención de Accidentes [STCONAPRA]. (2017). Modelo de atención médica prehospitalaria. México: Secretaría de Salud/Secretariado Técnico del Consejo Nacional para la Prevención de Accidentes.

Silva, A. E., Domínguez, A. J., Cortés, G., Castorena, A. y Polo, M. (2015). Análisis de satisfacción de universitarios mediante la minería de datos. RIDE Revista Iberoamericana para la Investigación y el Desarrollo Educativo, 5(10).

Surdez, E. G., Sandoval, M. del C. y Lamoyi, C. L. (2018). Satisfacción estudiantil en la valoración de la calidad educativa universitaria. Educación y Educadores, 21(1), 926. Recuperado de https://doi.org/10.5294/edu.2018.21.1.1. 